\title{
Two inequalities: a geometric and a combinatorial
}

\author{
Darko Veljan \\ Department of Mathematics, University of Zagreb, \\ Bijenička cesta 30, 10000 Zagreb, Croatia, \\ darko.veljan@gmail.com
}

\begin{abstract}
We present two interesting inequalities: one geometric and one combinatorial. The geometric one involves symmetric functions of side lengths of a triangle. It simultaneously improves Euler's inequality and isoperimetric inequality for triangles and has non-Euclidean versions. As a consequence, in combinatorics we apply it to degenerate (Fibonacci) triangles. We discuss similar inequalities for simplices in higher dimensions. The combinatorial inequality deals with the following question. What is more probable among maps: an injection or a surjection? For maps between finite sets, the answer is surjection. We present several proofs and provide a brief discussion on open problems for continuous maps for metric and other spaces.
\end{abstract}

Keywords: triangle inequality, tetrahedron and volume inequality, Euler's inequality in $2 \mathrm{D}$ and $3 \mathrm{D}$, combinatorial inequality, injective proof

MSC: 51M04, 51M09, 51M16, 05A20, 60C05

\section{Introduction}

The paper has two separate parts. The first part contains sections 1-4, and deals with geometric symmetric functions-inequalities for triangles and simplices; the second part is section 5, devoted to a combinatorial inequality which answers an intriguing question: what is more probable - surjections or injections?

DOI: https://doi.org/10.5592/CO/CCD.2018.11 


\section{Symmetric functions-inequality for side lengths of a triangle}

We shall prove an interesting and somewhat unusual inequality for side lengths of a triangle. It is symmetric in all three sides. Therefore, it can better be comprehended in terms of symmetric functions in three variables.

In standard notations, let $a, b$ and $c$ be side lengths of a triangle (even degenerate) and let $e_{1}=a+b+c, e_{2}=a b+b c+c a, e_{3}=a b c$ be the elementary symmetric functions of $a, b$ and $c$. Then the following symmetric inequality in $a, b, c$ holds.

\section{Theorem 1.1}

$$
e_{1}^{6}+12 e_{1}^{3} e_{3}+12 e_{1}^{2} e_{2}^{2}+36 e_{3}^{2} \geq 7 e_{1}^{4} e_{2}+40 e_{1} e_{2} e_{3} .
$$

Equality holds if and only if the triangle is equilateral, $a=b=c$.

Proof .

Let $S, 2 s, R$ and $r$ be the area, perimeter, circumradius and inradius, respectively, of the triangle with side lengths $a, b$ and $c$. Then we have

$$
\begin{aligned}
\frac{R}{r} & =\frac{a b c s}{4 S^{2}}=\frac{a b c}{4(s-a)(s-b)(s-c)}=\frac{2 a b c}{(-a+b+c)(a-b+c)(a+b-c)} \\
& \geq \frac{a b c+a^{3}+b^{3}+c^{3}}{2 a b c} \geq 2 .
\end{aligned}
$$

The second inequality in (2) is the AM-GM inequality for $a^{3}, b^{3}$ and $c^{3}$, and the first inequality is proved below. Note that it is an improvement of Euler's inequality $R / r \geq 2$ from 1765. For more details see [7], and for more on AM-GM inequality see $[8]$.

To prove the first inequality in (2), let $x, y$ and $z$ be the tangent segments from vertices to the incircle, so $a=y+z, b=z+x$, and $c=x+y$. Then it is easy to see that the first inequality in (2) is equivalent to

$$
\begin{aligned}
2 x^{2} y^{2}(x-z)(y-z) & +2 y^{2} z^{2}(y-x)(z-x)+2 z^{2} x^{2}(x-y)(z-y) \\
& +x^{4}(y-z)^{2}+y^{4}(z-x)^{2}+z^{4}(x-y)^{2} \geq 0 .
\end{aligned}
$$

To prove (3) it suffices to prove that the sum of the first three summands in (3) is non negative. Without loss of generality, we may assume that $x \leq y \leq z$. Then the half of the sum of the first three summands in (3) is greater or equal than 
$x^{2} y^{2}(x-z)(y-z)+z^{2} x^{2}(y-z)(x-y)+z^{2} x^{2}(z-y)(x-y)=x^{2} y^{2}(x-z)(y-z)$, and this number is greater or equal than 0 . This proves (3). The first inequality in (2) has on both sides fractions of symmetric functions in $a, b, c$. By expressing numerators and denominators in terms of $e_{1}, e_{2}$ and $e_{3}$, it is not hard to show that this is equivalent to the inequality (1). Finally, since the equality $R / r=2$ holds if and only if the triangle is equilateral, it follows that equality in (1) holds if and only if $a=b=c$.

We can write (2) also in the form

$$
\frac{R}{r}=\frac{(a+b+c) a b c}{8 S^{2}} \geq \frac{a b c+a^{3}+b^{3}+c^{3}}{2 a b c} .
$$

From Heron's formula

$$
16 S^{2}=e_{1}\left(4 e_{1} e_{2}-e_{1}^{3}-8 e_{3}\right),
$$

we thus obtain the following inequality equivalent to (1):

$$
4 S^{2}\left(e_{1}^{3}-3 e_{1} e_{2}+4 e_{3}\right) \leq e_{1} e_{3}^{2} .
$$

Equality in (4) again occurs if and only if the triangle is equilateral. The standard isoperimetric inequality for triangles reads as follows

$$
S \leq e_{1}^{2} \frac{\sqrt{3}}{36},
$$

with equality if and only if the triangle is equilateral. By comparing (4) and (5), we shall show that (4), which is equivalent to (1), in fact improves (5). Namely,

$$
4 S^{2}=e_{1}\left(4 e_{1} e_{2}-e_{1}^{3}-8 e_{3}\right) / 4 \leq e_{1} e_{3}^{2} /\left(e_{1}^{3}-3 e_{1} e_{2}+4 e_{3}\right) \leq e_{1}^{4} / 2^{2} 3^{3} .
$$

Here, the equality is Heron's formula, the first inequality is (1), and the second inequality is equivalent to

$$
e_{1}^{3}\left(e_{1}^{3}+4 e_{3}\right) \geq 3\left(e_{1}^{4} e_{2}+36 e_{3}^{2}\right) .
$$

But this follows by applying Newton's inequalities (see [8]), once as $e_{1}^{2} \geq 3 e_{2}$, and once as $e_{1}^{3} \geq 27 e_{3}$. So, we have proved the following.

Theorem 1.2 The inequality (1) improves not only Euler's inequality that the circumcircle of a triangle is at least twice longer than its incircle, but also improves the standard isoperimetric inequality (5) for triangles. This improvement becomes equality if and only if the triangle is equilateral. 
We can also give a lower bound for the area $S$. Again we start with Heron's formula written as

$$
S^{2}=s\left\{[(s-a)(s-b)(s-c)]^{1 / 3}\right\}^{3},
$$

and apply the geometric-harmonic inequalities to the last three factors of the previous expression to obtain

$$
S^{2} \geq s\left\{\frac{3(s-a)(s-b)(s-c)}{(s-a)(s-b)+(s-b)(s-c)+(s-a)(s-c)}\right\}^{3} .
$$

Now we express both the numerator and denominator in terms of $e_{1}, e_{2}$ and $e_{3}$. Then we can summarize inequalities (4), (5), (6) and (7) in the following chain of inequalities.

Theorem 1.3 The squared area $S^{2}$ of a triangle is bounded in terms of $e_{i}$ 's of side lengths as

$$
\left(27 e_{1} / 4\right)\left[\left(4 e_{1} e_{2}-e_{1}^{3}-8 e_{3}\right) /\left(4 e_{2}-e_{1}^{2}\right)\right]^{3} \leq 4 S^{2} \leq e_{1} e_{3}^{2} /\left(e_{1}^{3}-3 e_{1} e_{2}+4 e_{3}\right) \leq e_{1}^{4} / 108 .
$$

\section{Symmetric functions-inequalities for non-Euclidean tri- angles}

The spherical and hyperbolic versions of Euler's inequality $R / r \geq 2$, respectively, are the following inequalities (see [6]):

$$
\tan (R) / \tan (r) \geq 2 \text { and } \tanh (R) / \tanh (r) \geq 2 .
$$

As proved in [2], non-Euclidean Euler's inequalities (8) can be strengthened in a symmetric way via side-lengths, but not in the sense analogous to (2). It seems (2) is too strong in these cases. Still, improvements to non-Euclidean cases can be done by using the following Lemma proved in [2].

Lemma 2.1 If $f(a, b, c) \geq 0$ is an inequality which holds for all Euclidean triangles with side lengths $a, b, c$, then $f(s(a), s(b), s(c)) \geq 0$ for all spherical or hyperbolic triangles with side lengths $a, b, c$, where $s(x)=x / 2$ in Euclidean geometry, $s(x)=$ $\sin (x / 2)$ in spherical geometry and $s(x)=\sinh (x / 2)$ in hyperbolic geometry.

By using the above Lemma and Theorem 1 we conclude that the following theorem holds true. 
Theorem 2.2 Inequality (1) holds also for non-Euclidean triangles with side lengths $a, b$ and $c$ but with symmetric functions in corresponding quantities $s(a), s(b)$ and $s(c)$ as in Lemma. Equalities hold again if and only if $a=b=c$. These inequalities are simultaneous improvements of Euler's inequalities (8) and isoperimetric inequalities as (5) in both spherical and hyperbolic geometry.

\section{Degenerate triangles and Fibonacci numbers}

Theorem 1 and all of its equivalent forms hold also in the case of a degenerate triangle, for instance if $a+b=c$. A natural example of such a degenerate triangle is given by a Fibonacci triple $\left(F_{n-1}, F_{n}, F_{n+1}\right)$. Recall Heron's formula in the form

$$
16 S^{2}=\left(a^{2}+b^{2}+c^{2}\right)^{2}-2\left(a^{4}+b^{4}+c^{4}\right) .
$$

Since degenerate triangle has area $S=0$, it follows that the equality $a+b=c$ can be replaced by the totally symmetric expression

$$
\left(a^{2}+b^{2}+c^{2}\right)^{2}=2\left(a^{4}+b^{4}+c^{4}\right),
$$

known as Candido's identity (from 1950). In fact, it was first noted just for Fibonacci numbers. However, it is a pure algebraic result which holds in any commutative ring for any of its two elements $a, b$, and their sum $c$. This can easily be checked directly.

A little historical remark on Fibonacci numbers is due to M. Bhargava. He said in an interview that they should actually be called Virahanka-Fibonacci numbers after Indian mathematician Virahanka who discovered them back in 7th century, in the sense that $F_{n+1}$ is the number of ways to write $n$ as an ordered sum (composition) of 1's and 2's. On different aspects of Candido's identity see in [9].

Not only that Candido's identity (9) and inequality (1) hold for Fibonacci numbers but they also hold for any combinatorially interesting numbers satisfying recurrence of the form $c=a+b$. Examples include binomial coefficients (due to Pascal's formula), Padovan numbers $\left(P_{n}\right)$ defined by $P_{0}=P_{1}=P_{2}=1$ and $P_{n+2}=P_{n}+P_{n-1}$, where $P_{n}$ is the number of ways to write $n+2$ as an ordered sum of 2's and 3's, and many other. The inequality (1) in the degenerate case $a+b=c$ is equivalent to the trivial inequality $a^{2} b^{2} \geq 0$, for any two real numbers $a$, $b$, which reveals the fact that a square of any real number is nonnegative and the product of such numbers is also nonnegative. 


\section{Inequalities for tetrahedra and simplices}

Let $T=A B C D$ be a tetrahedron or a 3-dimensional simplex with edge lengths $a$, $b, c, a^{\prime}, b^{\prime}, c^{\prime}$, where $a, b, c$ form a triangle, $a$ is opposite to $a^{\prime}$ etc. Let $V=\operatorname{vol}(T)$ be the volume, $S$ the surface area of $T$, and $R$, and $r$, respectively, the circumradius and inradius of $T$, and $C$ the area of the Crelle triangle of $T$ whose side lengths are products $a a^{\prime}, b b^{\prime}, c c^{\prime}$ of opposite edge lengths of $T$. The Crelle formula $C=6 R V$, the fact $3 V=r S$, and Euler's inequality $R / r \geq 3$ imply

$$
54 V^{2} \leq C \cdot S
$$

By applying the standard isoperimetric inequality to both $C$ and $S$, from (10) we infer

$$
25 \cdot 36 V^{2} \leq\left(a a^{\prime}+b b^{\prime} c c^{\prime}\right)^{2}\left[(a+b+c)^{2}+\left(a+b^{\prime}+c^{\prime}\right)^{2}+\left(a^{\prime}+b+c^{\prime}\right)^{2}+\left(a^{\prime}+b^{\prime}+c\right)^{2}\right] .
$$

Substituting $V^{2}$ in (11) in terms of $a, a^{\prime}, \ldots, c^{\prime}$, for instance Euler's formula (from 1752):

$$
\begin{aligned}
144 V^{2}= & \left(2 a^{\prime} b^{\prime} c^{\prime}\right)^{2} \\
& -a^{\prime 2}\left(b^{\prime 2}+c^{\prime 2}-a^{2}\right)^{2}-b^{\prime 2}\left(c^{\prime 2}+a^{\prime 2}-b^{2}\right)^{2}-c^{\prime 2}\left(a^{\prime 2}+b^{\prime 2}-c^{2}\right)^{2} \\
& +\left(a^{\prime 2}+b^{\prime 2}-c^{2}\right)\left(b^{\prime 2}+c^{\prime 2}-a^{2}\right)\left(c^{\prime 2}+a^{\prime 2}-b^{2}\right),
\end{aligned}
$$

we get an inequality of degree 6 for edge lengths of $T$, in a way analogous to (1) for a triangle, with equality if $T$ is regular. However, (11) is not as sharp as the inequality

$$
72 V^{2} \leq a a^{\prime} b b^{\prime} c c^{\prime}
$$

with equality if and only if $T$ is regular (see [10],[11]). Inequality (11) can be improved by applying (4) to $C^{2}$ and all four face areas $S_{i}^{2}$, with $S=S_{1}+S_{2}+S_{3}+S_{4}$ and using the arithmetic-quadratic inequality for $S_{i}$ in the form $S^{2} \leq 4\left(S_{1}^{2}+S_{2}^{2}+\right.$ $\left.S_{3}^{2}+S_{4}^{2}\right)$. The obtained inequality becomes equality if and only if $T$ is equifacial, i.e. $S_{1}=S_{2}=S_{3}=S_{4}$, or equivalently $a=a^{\prime}, b=b^{\prime}, c=c^{\prime}$. It is in the range of the known inequality (see [4])

$$
72 V^{2} \leq\left(a a^{\prime}+b b^{\prime}-c c^{\prime}\right)\left(b b^{\prime}+c c^{\prime}-a a^{\prime}\right)\left(c c^{\prime}+a a^{\prime}-b b^{\prime}\right),
$$

with equality if and only if $T$ is equifacial.

Let us briefly explain Euler's inequality $R / r \geq 3$ for a tetrahedron $T$. Let $T^{\prime}$ be the tetrahedron whose vertices are centroids of faces of $T$. Then $T^{\prime}$ is similar to $T$ 
with the similarity coefficient 3 . Hence, the circumradius $R^{\prime}$ of $T^{\prime}$ is one third of $R$, that is, $R=3 R^{\prime}$. But, $R^{\prime} \geq r$, because the smallest ball that touches all faces of $T$ is just the inscribed ball of $T$. So, $R \geq 3 r$. The equality is attained if and only if $T$ is regular. The same argument works for any $n$-dimensional simplex (and of course, $R \geq n r$ in that case).

Unlike this simple argument, there is no evident argument for yet another inequality relating $R, r$ and the distance $d$ between the circumcenter and incenter of a tetrahedron. It is known as Grace-Danielsson's inequality (from 1949). It reads as follows

$$
d^{2}+(2 r)^{2} \leq(R-r)^{2}
$$

Euler's formula for triangles $d^{2}+r^{2}=(R-r)^{2}$ can easily be proved via inversion, but for Grace-Danielsson's inequality, a short elegant proof in the sense of CEEG (Classical Euclidean Elementary Geometry) is still missing. There is a proof using quantum information theory [5] and computer aided proof [3]. Corresponding formulas or inequalities for simplices in higher dimensions are also not known as well as non-Euclidean versions.

Recall that for triangles we found a rational non-constant symmetric function $f(a, b, c)$ such that (see $(2))$ :

$$
\frac{R}{r} \geq f(a, b, c) \geq 2,
$$

and this essentially refined Euler's inequality. A similar problem is for tetrahedron $T$ : find a non-constant simple enough symmetric function $\Theta$ in edge lengths $a, a^{\prime}, \ldots, c^{\prime}$ and in $a a^{\prime}, b b^{\prime}, c c^{\prime}$ such that

$$
\frac{R}{r}=\frac{C \cdot S}{18 V^{2}} \geq \Theta \geq 3 .
$$

We can express $C, S$ and $V$ in terms of edges of $T$ and get the corresponding inequality for edges of $T$, but this is not quite adequate because we have sum of four square roots, so it is not good in the above sense. In fact, in [7] we proved an interpolation of $(R / r)^{2} \geq 9$ in terms of symmetric functions of $a a^{\prime}, b b^{\prime}$ and $c c^{\prime}$.

For general simplices, besides exact volume formulas, the Cayley-Menger formula, some known inequalities may be of interest such as $([10],[11])$ :

$$
(n ! V)^{2} n^{n} \leq(n+1)^{n+1} R^{2 n}, \quad(n ! V)^{2} 2^{n} \leq(n+1)\left(\prod a_{i j}\right)^{4 /(n+1)} .
$$

Both of them attain equality if and only if the simplex is regular. For $n=2$, the right inequality (12) is not as good as our Theorem 1 (or equivalently, the inequality (4)). 
For $n=3$, we mentioned it earlier. For higher dimensions, no essential improvement of Euler's inequality is known.

\section{Are surjections more probable than injections? Yes - for finite sets!}

Problem 11957 of the American Mathematical Monthly (AMM) [12] was proposed by É. Pité, Paris, France, in vol. 124, February 2017. It is as follows (a bit rephrased).

Let $n \geq k \geq 1$ be integers. Prove that

$$
n^{k} S(n, k) \geq k^{n}\left(\begin{array}{l}
n \\
k
\end{array}\right)
$$

where $S(n, k)$ is the Stirling number of the second kind, equal to the number of partitions of an $n$-set into $k$ blocks (nonempty subsets).

We shall survey several proofs of (13) and provide some comments. Two solutions appeared in November 2018 issue of AMM ([13]) and one solution was published online earlier. This earlier solution by R. Tauraso, Rome, Italy, is by double induction on $n \geq k \geq 1$ using some known identities involving Stirling numbers and binomial coefficients and using convexity proving that the second derivative of the function $f_{k}(x)=(1+x / k)^{-k}$ is positive for $x>0$. We skip this proof and concentrate on combinatorial proofs.

But first some comments. For $k=1$ or $k=n$, the inequality (13) becomes equality, as well as for $k>n$, when $S(n, k)=\left(\begin{array}{l}n \\ k\end{array}\right)=0$. For $k=2, S(n, 2)=2^{n-1}-1$, so (13) reduces to the well known inequality $2^{n-1} \geq n$. For $k=n-1$, since $S(n, n-1)=\left(\begin{array}{l}n \\ 2\end{array}\right),(13)$ reduces to the also well known inequality $n^{n-1} \geq 2(n-1)^{n-1}$.

In fact, (13) is not very surprising, because $S(n, k)$ for large $n$ behaves as $k^{n} / k !$, so (13) is then of the type

$$
n^{k} \geq n^{\underline{k}}=n(n-1)(n-2) \ldots(n-k+1),
$$

which is obviously true.

Let us first prove a weaker inequality (interesting by itself):

$$
n^{k} S(n, k) \geq k^{n-k}\left(\begin{array}{l}
n \\
k
\end{array}\right) .
$$

Proof was given by M. Wildon, London, UK, in mathoverflow on 4 July 2017. (Recall, $[n]=\{1,2, \ldots, n\}$.) 
The right-hand side of (14) counts the set

$$
Y=\{(K, f) \mid K \subseteq[n] \text { is a } k \text { - set, and } f:[n] \backslash K \rightarrow K \text { is a function }\},
$$

while the left-hand side of (14) counts the set

$$
X=\{(P, g) \mid P \text { is a } k-\text { partition of }[n] \text {, and } g: P \rightarrow[n] \text { a function }\} .
$$

Define the function $h: Y \rightarrow X$ by $h(K, f)=(P, g)$, where $P=\left\{\{x\} \cup f^{-1}(x) \mid x \in\right.$ $K\}$, and $g\left(x \cup f^{-1}(x)\right)=x$. Function $g$ essentially picks a point (element) from each block, and serves to uniquely reconstruct the subset $K$ and the function $f$ : $[n] \backslash K \rightarrow K$. Hence, $h$ is an injection which proves (14).

One more (even simpler) proof of (14) is this. Let $H: X \rightarrow Y$, given by $H(P, g)=(K, f)$ be defined as follows. Let $P=\left\{B_{1}, \ldots, B_{k}\right\}$ and $g: P \rightarrow[n]$. Then let $K=\left\{x_{1}, \ldots, x_{k}\right\}, x_{i} \in B_{i}, i=1, \ldots, k$, be any selection of one element from each block of $P$ and define $f:[n] \backslash K \rightarrow K$. Let $x \in[n] \backslash K$, specifically $x \in B_{i} \backslash x_{i}$ and assume $g\left(B_{i}\right) \in B_{j} \subseteq[n]$. Then let $f(x):=x_{j}$. The map $H$ is clearly surjective, proving (14). Perhaps (13) can also be proved in a similar manner.

We now prove (13). The following elegant proof was given by T. Horine, Indiana Univ. SE, New Albany, IN. To partition the set $[n]$ into $k$ blocks first choose $k$ elements (points) and place one in each block. This choice can be done in $\left(\begin{array}{l}n \\ k\end{array}\right)$ ways. The rest, that is $n-k$ elements, can be put to those $k$ blocks in $k^{n-k}$ ways to complete a partition. But a partition with block sizes $s_{1}, \ldots, s_{k}$ has been counted $s_{1} s_{2} \ldots s_{k}$ times, since each block can be initiated by any of its $s_{i}$ elements. In each case, $s_{1}+\ldots+s_{k}=n$. By the AM-GM inequality $s_{1} s_{2} \ldots s_{k} \leq(n / k)^{k}$ for each partition. Hence we have

$$
(n / k)^{k} S(n, k)=\left[\left(s_{1}+\ldots+s_{k}\right) / k\right]^{k} S(n, k) \geq\left(s_{1} \ldots s_{k}\right) S(n, k) \geq\left(\begin{array}{l}
n \\
k
\end{array}\right) k^{n-k} .
$$

This proves (13).

A similar proof of (13) was provided by Filip Nikšić (Germany) in mathoverflow on 4 July 2017.

Given a $k$-partition $P$ of $[n]$ and a $k$-set $S$ of $[n]$, we say that $P$ splits $S$ if every block of $P$ contains exactly one point of $S$, that is, $B \cap S \neq \emptyset$ for every $B \in P$. We prove equivalent inequality to (13):

$$
k^{n-k}\left(\begin{array}{l}
n \\
k
\end{array}\right) \leq(n / k)^{k} S(n, k) .
$$


Let $M$ be a $(0,1)$-matrix whose rows are indexed by $k$-partitions $P$ of $[n]$ and columns by $k$-subsets $S$ of $[n]$. (So, $M$ is of the size $S(n, k) \times\left(\begin{array}{l}n \\ k\end{array}\right)$.) The entry $M(P, S)=1$ if and only if $P$ splits $S$.

We count the number of ones in two ways. The number of ones in a column indexed by $S$ is the number of partitions that split $S$. Such a partition is uniquely determined by a map $[n] \backslash S \rightarrow S$ that maps $x \in[n] \backslash S$ to $y \in S$ if $x$ and $y$ are in the same block of the partition. Hence the number of ones in the column is $k^{n-k}$ and the total number of ones in $M$ is $k^{n-k}\left(\begin{array}{l}n \\ k\end{array}\right)$. On the other hand, the number of ones in a row indexed by $P=\left\{B_{1}, \ldots, B_{k}\right\}$ is the number of $k$-sets split by $P$. Such a set is uniquely determined by a choice of one element from each block. Hence, the number of ones in the row is $s_{1} s_{2} \ldots s_{k}$, where $s_{i}=\left|B_{i}\right|, i=1, \ldots, k$. The total number of ones in $M$ is therefore the sum of such products over all $k$-partitions of $[n]$. By the AM-GM inequality (as in the previous proof) we have $s_{1} s_{2} \ldots s_{k} \leq(n / k)^{k}$. Finally, we conclude

$$
k^{n-k}\left(\begin{array}{l}
n \\
k
\end{array}\right)=\sum s_{1} s_{2} \ldots s_{k} \leq(n / k)^{k} S(n, k) .
$$

This proves (15), and hence (13).

One more combinatorial proof of (13) was provided by M. Wildon. By considering $|\operatorname{Im}(f)|=r$ and $|\operatorname{Im}(g)|=r \leq k$, for various $r$, (13) reduces to show the following interesting inequality

$$
S(n, k) S(k, r) \geq\left(\begin{array}{l}
n-r \\
k-r
\end{array}\right) S(n, r)
$$

for all $r \leq k \leq n$.

To prove this inequality, start with given $r$-partition $Q$ of $[n]$. Let $M(Q)$ be the $r$-subset of $[n]$ consisting of the largest element in each block of $Q$. Choose also a set $T$ of $k-r$ elements in the set $[n] \backslash M(Q)$. The right-hand side counts all such pairs $(Q, T)$.

Given such a pair $(Q, T)$, define a $k$-partition $P$ of $[n]$ that refines $Q$. Simply extract each element of $T$ from its block in $Q$ and make a new singleton block.

Next consider all pairs $(Q, T)$, where $Q$ is an $r$-partition and $P$ a $k$-partition of $[n]$ and $P$ a refinement of $Q$. We can build such pairs by first choosing $P$ and then grouping the blocks of $P$ into a partition with $r$ blocks. Hence, there are $S(n, k) S(k, r)$ such pairs. So, it suffices to show that the map $(Q, T) \mapsto(Q, P)$ is $1-1$.

If $(Q, P)$ arises from $(Q, T)$ by this map, then $P$ has at least $k-r$ singleton blocks. The element $x$ of a singleton block lies in $T$ if and only if $x \notin M(Q)$. Thus 
we can uniquely reconstruct $(Q, T)$ from $(Q, P)$ and the map is injective as desired.

Finally, let us explain the title of this paragraph. Namely, the main inequality (13) is equivalent to

$$
k ! S(n, k) / k^{n} \geq n^{\underline{k}} / n^{k} .
$$

The right-hand side of (16) is the probability that a uniformly chosen random function $[k] \rightarrow[n]$ is injective, while the left-hand side of (16) is the probability that a uniformly chosen random function $[n] \rightarrow[k]$ is surjective. So, surjections are more probable than injections among maps between finite sets.

Small numerical examples of (16): for $n=7$ and $k=4, \operatorname{Prob}([7] \rightarrow[4] \operatorname{surj})=$ $0.51269 \ldots \geq 0.34985 \ldots=\operatorname{Prob}([4] \rightarrow[7] i n j)$, for $n=4$ and $k=3, \operatorname{Prob}([4] \rightarrow$ $[3] \operatorname{surj})=4 / 9 \approx 0.44 \geq 0.375=\operatorname{Prob}([3] \rightarrow[4] i n j)$. So, the event that a uniformly chosen random map [4] $\rightarrow$ [3] is surjective is not very likely to happen, because its probability is less than 0.5 .

Note that for fixed $k$, both sides of (16) tend to 1 , when $n \rightarrow \infty$. But for $k=n$, or $k=n-1$, both sides of (16) tend to 0 , when $n \rightarrow \infty$.

Finally, let us make some comments and raise some problems. It would be interesting to find a direct probabilistic argument for (16). Next, what is a "continuous" version of (16)?

For instance, if $X$ and $Y$ are compact metric spaces and, say, $Y \subseteq X$, is it more probable that a uniformly and randomly chosen (continuous) map $X \rightarrow Y$ is surjective (covering) than a uniformly and randomly chosen (continuous) map $Y \rightarrow X$ injective (embedding)? Of course, this requires a choice of an appropriate probability measure (i.e. a positive regular Borel measure $m$ with $m(X)=1$ and if $f \in L^{1}(X, m)$, then $m(f)=\int f d m$, and similarly on $Y$ ). A related but more complex problem is to find probability distribution of injections among all maps $Y \rightarrow X$ (and similarly for surjections among all maps $X \rightarrow Y$ ), or at least find good bounds. We can restrict questions to, say, manifolds (Riemannian, smooth or topological), or generalize further to more general (topological) spaces or even to more general categorical framework. Even in the case $X=Y=[0,1]$ nothing essential in this respect is known. Another "concrete" examples are: $Y=I=[0,1]$, and $X=I^{3}$ ( a sort of self-avoiding walk problem) and $Y=S^{1}$ (circle) and $X=S^{3}$ (3-sphere), problems in knot theory. Perhaps a good starting point to think about such general problems is Grothendieck's inequality (see e.g. [1]). 


\section{References}

[1] S. Friedland, L.-H. Lim and J. Zhang, An elementary and unified proof of Grothendieck's inequality, online 29 Dec 2018.

[2] R. Guo, E. Black and C. Smith, Strengthened Euler's inequality in spherical and hyperbolic geometries, arXiv: 1704.05373 [math.MG], 17 Apr 2017.

[3] L. László, On the Grace-Danielsson inequality for tetrahedra, Discrete Appl. Math. 256 (2019) 83-90.

[4] M. Mazur, An inequality for the volume of a tetrahedron, Amer. Math. Monthly 125 (2018) 273-275.

[5] A. Milne, The Euler and Grace-Danielsson inequalities for nested triangles and tetrahedra: a derivation and generalization using quantum information theory, J. Geom. 106 (2015) 455-463.

[6] D. Svrtan and D. Veljan, Non Euclidean versions of some classical triangle inequalities, Forum Geom. 12 (2012) 197-209.

[7] D. Veljan, Symmetric functions-inequalities for triangles and simplices: Euler's case, submitted.

[8] D. Veljan, The AM-GM inequality from different viewpoints, Elem. Math. 72 (2017) 24-34.

[9] D. Veljan, A note on Candido's identity and Heron's formula, Proceedings of the $1^{\text {st }}$ Croatian Combinatorial. Days, Zagreb, Sept. 29-30, 2016 (Eds. T. Došlić and I. Martinjak), Fac. Civil Eng., Univ. Zagreb, 2017, pp. 95-105.

[10] D. Veljan, The sine theorem and inequalities for volumes of simplices and determinants, Lin. Alg. Appl. 219 (1996) 79-91.

[11] V. Volenec, D. Veljan and J. Pečarić, Inequalities for volumes of simplices in terms of their faces, Geom. Dedicata 37 (1998), 57-62.

[12] Problem 11957, Amer. Math. Monthly 124 (2017) 179.

[13] Problem 11957, Amer. Math. Monthly 125 (2018) 858-859. 